\title{
Papers
}

\section{Using vital signs to diagnose impaired consciousness: cross sectional observational study}

\author{
Masayuki Ikeda, Takashi Matsunaga, Noritsugu Irabu, Shohji Yoshida
}

\begin{abstract}
Objectives To determine whether any vital signs can be used to quickly identify brain lesions in patients with impaired consciousness.

Design Cross sectional observational study. Setting Emergency department of an urban hospital, Japan.

Participants 529 consecutive patients (mean age 65 years) presenting with impaired consciousness (score $<15$ on the Glasgow coma scale) during 2000.

Main outcome measures The receiver operating characteristic curve was used to quantify the relation between the vital signs on arrival and the final diagnosis of a brain lesion. Stratum specific likelihood ratios were calculated to define strata with optimal discriminating power.

Results 312 (59\%) had a brain lesion which accounted for the impaired consciousness. The area under the receiver operating curve for systolic blood pressure was 0.90 (SE 0.01), indicating significantly higher accuracy $(\mathrm{P}<0.01)$ in the identification of a brain lesion than using diastolic pressure $0.82(0.02)$ or pulse rate $0.63(0.03)$. Likelihood ratios for systolic blood pressure lower than $90 \mathrm{~mm} \mathrm{Hg}$ were less than 0.04 , and those for systolic pressure higher than 170 $\mathrm{mm} \mathrm{Hg}$ were greater than 6.09.

Conclusions Systolic blood pressure is useful for diagnosing brain lesions in patients with impaired consciousness.
\end{abstract}

\section{Introduction}

Diagnosing impaired consciousness is always a challenge. In many situations in the emergency room, doctors have to save time by doing other examinations first-for example, for hypoglycaemic coma, drug poisoning, and hepatic encaephalopathy. As doctors are not prepared to risk missing an abnormality, ${ }^{2}$ the use of computed tomography to screen patients with impaired consciousness for a brain lesion has become routine. Computed tomography of the cranium visualises brain lesions, but it does not identify extrinsic or metabolic brain dysfunctions, which affect more than half of patients with impaired consciousness. ${ }^{1}$

Previous studies suggest that the vital signs-blood pressure and pulse rate-are useful diagnostic tools in patients with impaired consciousness. Patients with acute stroke and those with increased intracranial pressure often have hypertension. ${ }^{34}$ The Cushing response (bradycardia with hypertension) is a well recognised clinical manifestation of increased intracranial pressure. ${ }^{5}$ Hypotension and tachycardia, on the other hand, are usual in states of depressed consciousnessfor example, intoxication, endocrine diseases, and sepsis-because of metabolic brain dysfunction. ${ }^{6}$

The continuous scale of the vital signs is related to the receiver operating characteristic curve. ${ }^{78}$ These curves show how well a test performs by plotting the test's sensitivity against its false positive rate ( 1 - specificity) at different levels of positivity or negativity. ${ }^{78}$ Analysis of these curves is a preferred method for evaluating screening tests, with a continuous scale used to distinguish between patients with and without a disease.

We examined whether vital signs could be used to distinguish between patients with impaired consciousness who are likely to have an intracranial lesion from those who are not. We plotted receiver operating characteristic curves for the ability of each vital sign to identify patients with a brain lesion. Cut-off points were determined by calculating stratum specific likelihood ratios for the different levels of the vital signs. ${ }^{7}$ These ratios and their 95\% confidence intervals are a useful way of defining highly discriminating strata.

\section{Methods}

During 2000, we conducted a cross sectional observational study at the emergency department of Asahi General Hospital, Asahi, Chiba, Japan. This urban hospital primarily serves a general population and has about 15000 admissions each year. On arrival, every patient had their systolic and diastolic blood pressure, pulse rate, and body temperature measured, and a score on the Glasgow coma scale was awarded. ${ }^{9}$ We defined impaired consciousness as a score of $<15$ on the scale.

We prospectively evaluated 529 consecutive patients with impaired consciousness who were at least 15 years old. We excluded patients with head injury because this was obviously the cause of their impaired consciousness. To determine the cause of their impaired consciousness, we admitted all eligible patients to the ward and followed them up until discharge. We considered the final diagnosis accounting for the impaired consciousness as the reference diagnosis.

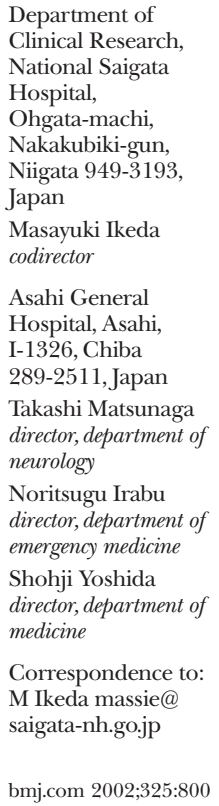

Correspondence to: M Ikeda massie@ saigata-nh.go.jp

bmj.com 2002;325:800 


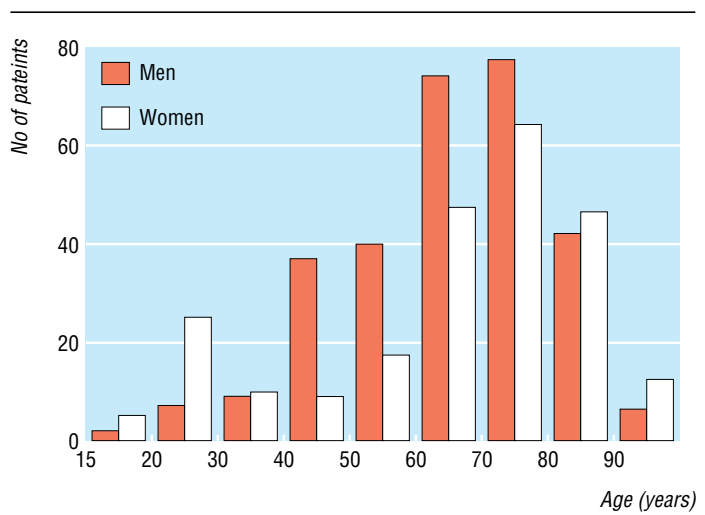

Fig 1 Age distribution of 529 patients with impaired consciousness

We did not use a standardised diagnostic protocol because we wanted to use the vital signs to identify patients with brain lesions, in a clinical setting, where the test might actually be used. The institutional review board approved the study and waived the requirement to obtain informed consent because we planned to record data ordinarily collected in the evaluation of patients.

\section{Statistical analysis}

We used the receiver operating characteristic curve to quantify how well the vital signs could be used to diagnose a brain lesion. ${ }^{78}$ Using a non-parametric method, we estimated the diagnostic accuracy of each vital sign by calculating the area under the curve and its standard error. $^{10}$ We also determined the significance of differences between areas under curves with a method described elsewhere. ${ }^{10}$

The stratum specific likelihood ratio (SSLR) is the probability of a given test result when the disease is present, divided by the probability of the same test result when the disease is absent. ${ }^{9}$ We determined these ratios by means of the formula $\operatorname{SSLR}=\left(\mathrm{x}_{1} / \mathrm{n}_{1}\right) /\left(\mathrm{x}_{0} / \mathrm{n}_{0}\right)$, where $x_{1}$ is the number of patients in the stratum with a brain lesion, $\mathrm{n}_{1}$ is the total number with a lesion, $\mathrm{x}_{0}$ is the number of patients in the stratum without a lesion, and $\mathrm{n}_{0}$ is the total number of patients without a lesion. We used the logit method to calculate the 95\% confidence intervals. ${ }^{7}$ All of the analysis was done by computer, with formulas that are published elsewhere. ${ }^{7}$

\section{Results}

\section{Patient population}

During 2000, we admitted 15293 patients of whom 529 (3.5\%; 235 women) had impaired consciousness of non-traumatic cause. The mean age was 65 years (range 15 to 97 years; SD 21 years). Table 1 shows the characteristics of the patients according to the presence $(n=312)$ or absence $(n=217)$ of a brain lesion.

Table 1 Characteristics of patients with impaired consciousness presenting during 2000

\begin{tabular}{lccc} 
& $\begin{array}{c}\text { With brain lesion } \\
(\mathbf{n}=\mathbf{3 1 2})\end{array}$ & $\begin{array}{c}\text { Without brain lesion } \\
(\mathbf{n}=\mathbf{2 1 7})\end{array}$ & P value \\
\hline Mean (SD) age (years) & $65.8(14.0)$ & $62.7(21.8)$ & $0.07 \dagger$ \\
\hline No (\%) of women & $137(43.9)$ & $98(45.2)$ & $0.78^{\star}$ \\
\hline Glasgow coma scale score (SD) & $9.88(3.79)$ & $9.49(3.55)$ & $0.23 \dagger$ \\
\hline
\end{tabular}

†Determined using Student's $t$ test.

${ }^{*}$ Determined using $\chi^{2}$ analysis.
The two groups did not differ significantly in age, sex, or score on the Glasgow coma scale. Figure 1 shows the age and sex distributions of the two groups. Most patients under the age of 70 years were men, apart from in the 20 s age group; more women in their $20 \mathrm{~s}$ attempt suicide by taking an overdose of psychotropic drugs. This age distribution is similar to those of previous studies on patients with impaired consciousness. ${ }^{311}$

\section{Vital signs in patients}

Table 2 shows the cause of impaired consciousness and the vital signs of the patients. Some of the vital signs clearly distinguish between the patients with brain lesions and those without. Of the $312(59 \%)$ patients with a brain lesion, $259(83 \%)$ had a final diagnosis of stroke, $29(9 \%)$ had epilepsy, 13 (\%) had a brain tumour, and $11(2.1 \%)$ had meningitis or encephalitis. Of the 217 (41\%) patients without a brain lesion, 95 $(18 \%)$ had diffuse hypoxia or ischaemia, $59(19 \%)$ had drug poisoning, 20 (64\%) had hepatic encephalopathy, $12(4 \%)$ had diabetic coma, and $11(4 \%)$ had ionic imbalance. Diffuse hypoxia or ischaemia includes
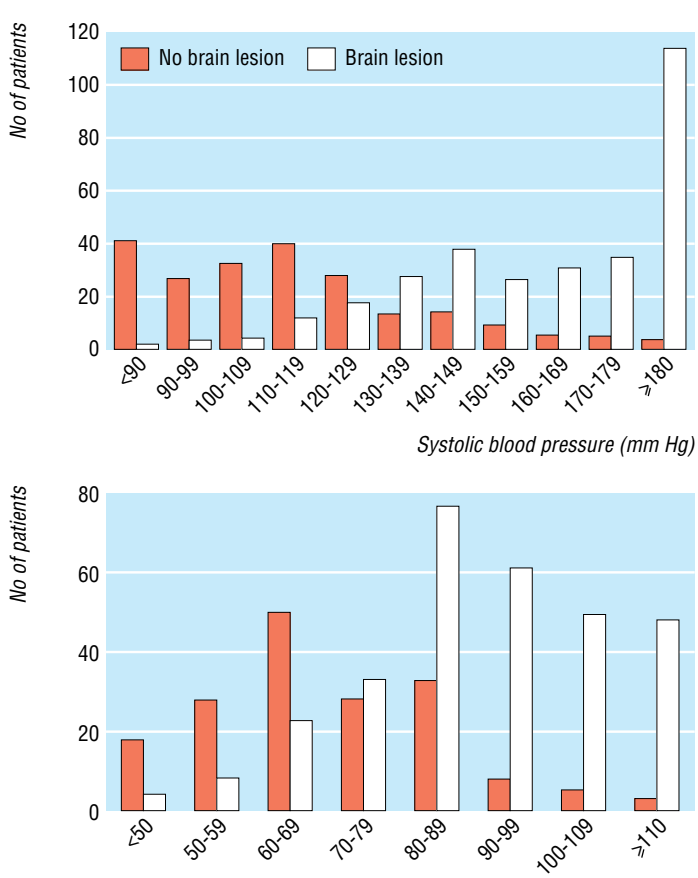

Diastolic blood pressure $(\mathrm{mm} \mathrm{Hg})$

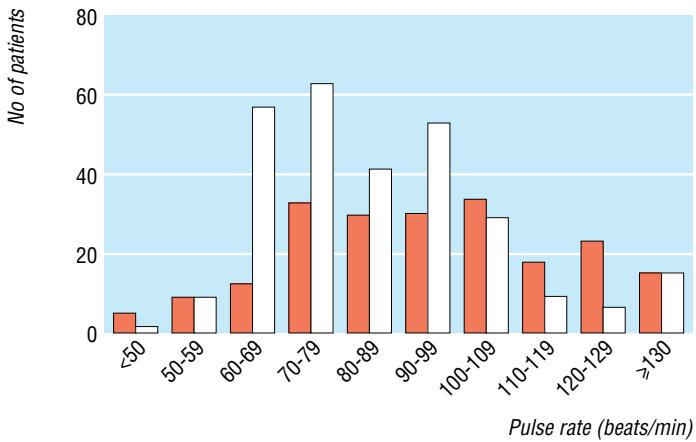

Fig 2 Distribution of systolic blood pressure (top), diastolic blood pressure (middle), and pulse rate (bottom) among patients with impaired consciousness 
Table 2 Cause of impaired consciousness and vital signs. Values in parentheses are standard deviations except where indicated

\begin{tabular}{|c|c|c|c|c|c|c|}
\hline & \multirow[b]{2}{*}{ No $(\%)$} & \multirow{2}{*}{$\begin{array}{l}\text { Glasgow coma } \\
\text { scale score }\end{array}$} & \multicolumn{2}{|c|}{ Blood pressure $(\mathrm{mm} \mathrm{Hg})$} & \multirow{2}{*}{$\begin{array}{l}\text { Pulse rate } \\
\text { (beats/min) }\end{array}$} & \multirow{2}{*}{$\begin{array}{l}\text { Body temperature } \\
\left({ }^{\circ} \mathrm{C}\right)\end{array}$} \\
\hline & & & Systolic & Diastolic & & \\
\hline \multicolumn{7}{|l|}{ With brain lesion } \\
\hline Stroke: & $259(49)$ & $10.3(3.7)$ & $172(35)$ & $92(19)$ & $81(18)$ & $36.5(0.9)$ \\
\hline Haemorrhage & $104(20)$ & $10.0(3.7)$ & $184(35)$ & $98(18)$ & $81(18)$ & $36.5(0.8)$ \\
\hline Infarction & $97(18)$ & $10.5(3.3)$ & $165(31)$ & $87(17)$ & $80(18)$ & $36.4(0.7)$ \\
\hline Subarachnoidal haemorrhage & $41(7.8)$ & $10.0(4.3)$ & $169(39)$ & $91(20)$ & $82(18)$ & $36.3(1.1)$ \\
\hline Subdural haematoma & $17(3.2)$ & $11.3(3.6)$ & $153(34)$ & $82(25)$ & $80(15)$ & $37.0(0.8)$ \\
\hline Brain tumour & $13(2.5)$ & $7.9(3.9)$ & $147(33)$ & $78(12)$ & $98(23)$ & $36.8(0.9)$ \\
\hline Epilepsy & $29(5.5)$ & $6.7(3.7)$ & $144(33)$ & $80(18)$ & $108(23)$ & $36.9(1.1)$ \\
\hline Meningitis or encephalitis & $11(2.1)$ & $11.8(1.9)$ & $146(34)$ & $88(15)$ & $95(21)$ & $37.9(1.2)$ \\
\hline Total & $312(59)$ & $9.9(3.8)$ & $168(36)^{*}$ & $90(19)^{*}$ & $84(20)^{\star}$ & $36.6(1.0)$ \\
\hline \multicolumn{7}{|l|}{ Without brain lesion } \\
\hline Drug poisoning & $59(11)$ & $9.0(3.9)$ & $109(21)$ & $67(15)$ & $87(18)$ & $35.8(2.8)$ \\
\hline Hepatic coma & $20(3.8)$ & $9.5(3.5)$ & $121(33)$ & $69(21)$ & $98(26)$ & $37.0(1.9)$ \\
\hline Diabetic coma & $12(2.3)$ & $11.7(2.3)$ & $116(21)$ & $74(12)$ & $103(22)$ & $36.2(2.1)$ \\
\hline Hypoglycaemia & $7(1.3)$ & $8.7(4.1)$ & $120(42)$ & $74(22)$ & $75(18)$ & $35.5(1.6)$ \\
\hline Ionic imbalance & $11(2.1)$ & $9.5(2.9)$ & $107(21)$ & $69(19)$ & $84(23)$ & $36.6(1.0)$ \\
\hline Uraemia & $3(0.6)$ & $11.0(4.4)$ & $137(40)$ & $65(21)$ & $81(29)$ & $35.7(1.0)$ \\
\hline Diffuse hypoxia or ischaemia $^{\dagger}$ & $95(18)$ & $9.6(3.5)$ & $110(28)$ & $66(17)$ & $100(26)$ & $37.5(1.5)$ \\
\hline Others $^{\ddagger}$ & $10(1.9)$ & $9.4(3.5)$ & $103(25)$ & $62(16)$ & $86(20)$ & $35.9(2.4)$ \\
\hline Total & $217(41)$ & $9.5(3.6)$ & $111(27)$ & $67(17)$ & $94(24)$ & $36.8(2.0)$ \\
\hline
\end{tabular}

\section{${ }^{\star} \mathrm{P}<0.0001$.}

†Includes encephalopathy through hypoxia, hypercapnia, and systemic illness such as sepsis.

¥0thers include Addison’s disease, hypothyroidism, neuroleptic malignant syndrome, catatonic stupor, thiamine deficiency, and anaphylactic shock.

encephalopathy due to hypoxia, hypercapnia, or systemic illness such as sepsis.

Compared with those patients without a lesion, those with a lesion had significantly higher systolic blood pressure (168 (SD 36) $\mathrm{mm} \mathrm{Hg} v 111$ (27) $\mathrm{mm}$ $\mathrm{Hg} ; \mathrm{P}<0.0001)$, higher diastolic blood pressure $(90$ (19) $\mathrm{mm} \mathrm{Hg} v 67$ (17) mm Hg; $\mathrm{P}<0.0001)$, and significantly lower pulse rate $(84$ (20) beats/min v 94 (24) beats/min; $\mathrm{P}<0.0001)$. Body temperature between the groups did not differ.

The large standard deviation for each vital sign indicates considerable overlap between the two groups: using the vital signs to diagnose brain lesions in clinical practice is difficult (fig 2). A clearer view of the diagnostic significance of the vital signs can be obtained by determining the sensitivity and specificity in different strata: we measured the area under the receiver operating characteristic curves and calculated stratum specific likelihood ratios.

Firstly, we analysed the diagnostic value of systolic and diastolic blood pressure and pulse rate with area under curves. Figure 3 shows the receiver operating characteristic curves for each vital sign. We found an area of 0.90 (SE 0.01) for systolic and $0.82(0.02)$ for diastolic blood pressure and 0.63 (0.03) for pulse. Systolic blood pressure provides the greatest sensitivity for all specificities. Accordingly, the curve area was significantly higher for systolic than for diastolic blood pressure $(\mathrm{z}=4.53, \mathrm{P}<0.01)$.

We calculated stratum specific likelihood ratios with $95 \%$ confidence intervals to assess the discriminating power of the strata used to plot each curve (table 3). For systolic blood pressure-which curve analysis showed was the most useful tool-we identified 11 strata. With the pretest probability of having a brain lesion of 0.59 (312 patients with a brain lesion out of a total of 529) in our study and Bayes' theorem, we estimated the post-test probability for each stratum. Because diastolic blood pressure had only eight strata and pulse rate had only 10 , they are of less diagnostic value.

\section{Discussion}

Systolic blood pressure is the best vital sign for distinguishing whether impaired consciousness is because of a brain lesion. Previous clinical batteries to determine the nature and site of a lesion impairing consciousness define individual criteria which are comprehensive but too complex for general use. ${ }^{11} 12$

The accuracy of the area under receiver operating curves is classified as low if the area is between 0.5 and 0.7; as moderate, if between 0.7 and 0.9 ; and as high, if greater than $0.9 .^{13}$ Judged by their areas, the accuracy of diastolic blood pressure (0.82) is moderate and that of systolic blood pressure (0.9), which is significantly

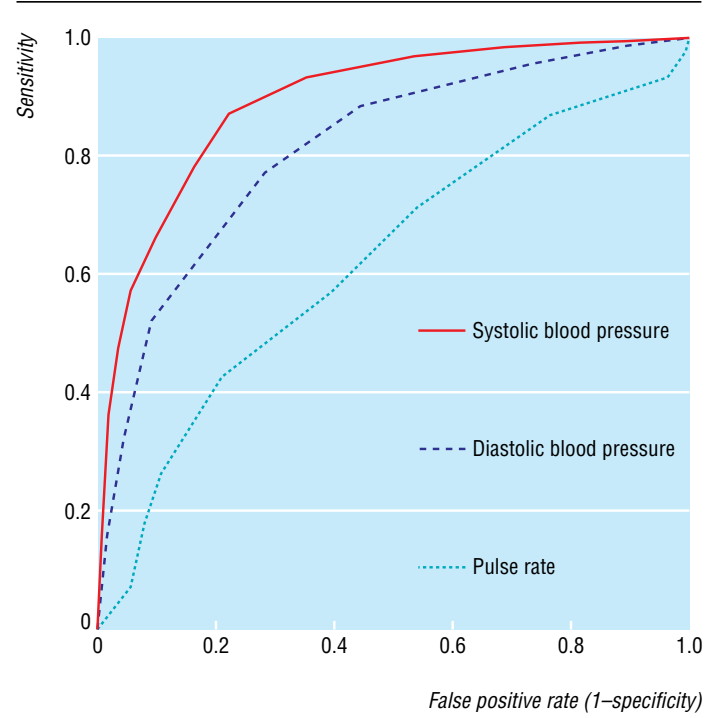

Fig 3 Sensitivity and false positive rate (1-specificity) for area under receiver operating characteristic curve for different strata of the vital signs, as given in table 3 
Table 3 Strata for receiver operating characteristic curves and stratum specific likelihood ratios

\begin{tabular}{|c|c|c|c|c|c|c|}
\hline \multirow[b]{2}{*}{ Strata } & \multicolumn{2}{|c|}{ No of patients } & \multirow[b]{2}{*}{ Sensitivity } & \multirow[b]{2}{*}{ Specificity } & \multirow{2}{*}{$\begin{array}{l}\text { Likelihood ratio } \\
(95 \% \mathrm{Cl})\end{array}$} & \multirow[b]{2}{*}{ Post-test probability } \\
\hline & Without brain lesion & With brain lesion & & & & \\
\hline \multicolumn{7}{|c|}{ Systolic blood pressure (mm Hg) } \\
\hline$<90$ & 41 & 2 & 0.99 & 0.19 & $0.03(0.01$ to 0.12$)$ & 0.04 \\
\hline 100-109 & 33 & 4 & 0.97 & 0.47 & $0.08(0.03$ to 0.22$)$ & 0.23 \\
\hline 110-119 & 40 & 12 & 0.93 & 0.65 & 0.21 (0.11 to 0.38$)$ & 0.39 \\
\hline 140-149 & 14 & 38 & 0.66 & 0.90 & 1.89 (1.06 to 3.37$)$ & 0.73 \\
\hline $150-159$ & 9 & 27 & 0.58 & 0.94 & 2.09 (1.02 to 4.27$)$ & 0.75 \\
\hline 160-169 & 5 & 31 & 0.48 & 0.97 & 4.31 (1.77 to 10.49$)$ & 0.86 \\
\hline 170-179 & 4 & 35 & 0.37 & 0.99 & 6.09 (2.32 to 15.98$)$ & 0.90 \\
\hline$\geqslant 180$ & 3 & 114 & 0 & 1.00 & 26.43 (9.25 to 75.48$)$ & 0.97 \\
\hline $70-79$ & 28 & 33 & 0.77 & 0.72 & 0.67 (0.42 to 1.07) & 0.49 \\
\hline $80-89$ & 33 & 76 & 0.52 & 0.91 & 1.31 (0.91 to 1.88$)$ & 0.65 \\
\hline $90-99$ & 8 & 61 & 0.32 & 0.96 & 4.34 (2.17 to 8.68 ) & 0.86 \\
\hline 100-109 & 5 & 49 & 0.16 & 0.99 & 5.58 (2.36 to 13.20$)$ & 0.89 \\
\hline$\geqslant 110$ & 2 & 48 & 0 & 1.00 & 13.67 (3.89 to 48.06$)$ & 0.95 \\
\hline \multicolumn{7}{|c|}{ Pulse rate (beats/min) } \\
\hline$<50$ & 5 & 2 & 0.98 & 0.01 & 3.38 (0.77 to 14.92$)$ & 0.70 \\
\hline 50-59 & 9 & 9 & 0.93 & 0.04 & 1.35 (0.56 to 3.27$)$ & 0.48 \\
\hline $60-69$ & 13 & 57 & 0.87 & 0.24 & 0.31 (0.18 to 0.54$)$ & 0.18 \\
\hline $70-79$ & 33 & 63 & 0.71 & 0.46 & 0.71 (0.49 to 1.03) & 0.33 \\
\hline
\end{tabular}

higher than that of diastolic blood pressure, is marginally high.

The classic Cushing response and previous studies on systemic response to impaired autoregulation of cerebral blood flow explain the increase in systemic blood pressure in patients with impaired consciousness caused by a brain lesion. ${ }^{5}$ In animal experiments, brain ischaemia results in an increase in systemic blood pressure. $^{14}{ }^{15}$ As many as $84 \%$ of patients with stroke have increased blood pressure in the acute phase. ${ }^{4}$ Hypertension can identify patients with impaired consciousness likely to have been caused by a stroke. ${ }^{3}$ In contrast, hypotension is usually associated with metabolic brain dysfunction caused by drug intoxication, sepsis, diabetic coma, or hepatic encephalopathy: systolic blood pressure should be a good measure to determine whether patients have a brain lesion. ${ }^{6}$

When computed tomography is available in an emergency department, clinicians order brain scans for almost every patient with impaired consciousness, but about half of patients with impaired consciousness, both in our work and Plum and Posner's, had no organic brain lesion. ${ }^{1}$ Perhaps some patients who are considered candidates for brain imaging can be classified as unlikely to have a brain lesion on clinical grounds. Our study indicates that systolic blood pressure can discriminate.

Our findings are limited, because of the characteristics of the patients we studied. Firstly, the older mean age, 65 years, than in previous studies, 54 years and 58 years, may be because of an older population in our

\section{What is already known on this topic}

Brain imaging and neurological examination of patients with impaired consciousness are often a waste of time and resources and can delay correct diagnosis

\section{What this paper adds}

Systolic blood pressure distinguishes patients with impaired consciousness who are at high risk from those who are at low risk of an organic brain lesion

General use of systolic blood pressure in the diagnosis of impaired consciousness may have clinical and economic benefits

local community. ${ }^{31}$ Secondly, the incidence of stroke in our study $(49 \%)$ was higher than in studies of Plum and Posner $(26 \% ; 130 / 500)$ and Yamashiro et al $(28 \%$; $49 / 175)$ but lower than among British patients with coma (57\%). ${ }^{1311}$ Thirdly, the high incidence of diffuse hypoxia or ischaemia $(18 \%)$ probably reflects the vulnerability of the older patients in our study to severe systemic infections-for example, pneumonia, pyelonephritis, and cholecystitis. Bates et al observed a similar high incidence of diffuse hypoxia or ischaemia among patients with coma. ${ }^{11}$

Our study confirms the validity of systolic blood pressure for discriminating between patients with 
impaired consciousness who have a brain lesion and those who do not. Using systolic blood pressure in the diagnosis of impaired consciousness should give both clinical and economic benefits.

We thank Toshiaki Furukawa for technical advice on the receiver operating characteristic curves and stratum specific likelihood ratios.

Contributors: MI designed the study, analysed the data, and wrote the report. TM and NI collected the data. SY helped prepare both the study and the report. MI is guarantor.

Funding: No additional funding.

Competing interests: None declared.

1 Plum F, Posner JB. The diagnosis of stupor and coma. 3rd ed. Philadelphia: FA Davis, 1980.

2 Graham ID, Stiell IG, Laupacis A, O'Connor AM, Wells GA. Emergency physicians' attitudes toward and use of clinical decision rules for radiography. Acad Emerg Med 1998;5:134-40.

3 Yamashiro S, Oda Y, Kanegae S, Shirahama M, Yoshihara K, Fukui T, et al. Informative usefulness of age, sex and vital signs in the differential diagnosis of disturbed consciousness among 175 emergency outpatient Fukuoka Igaku Zasshi 1994;85:353-60.

4 Wallace JD, Levy LL. Blood pressure after stroke. JAMA 1981;246: 2177-80.
5 Guyton AC, Hall JE. Textbook of medical physiology. 9th ed. Philadelphia: WB Sauders, 1996:209-20.

6 Victor M, Ropper AH. Coma and related disorders of consciouness. In: Adams and Victor's principles of neurology. 7th ed. New York: McGraw-Hill, 2001:366-89.

7 Peirce JC, Cornell RG. Integrating stratum-specific likelihood ratios with the analysis of ROC curves. Med Decis Making 1993;13:141-51.

8 Furukawa TA, Goldberg DP, Rabe Hesketh S, Ustun TB Stratum-specific likelihood ratios of two versions of the general health questionnaire. Psychol Med 2001;31:519-29.

9 Teasdale G, Jennett B. Assessment of coma and impaired consciousness: a practical scale. Lancet 1974;ii:81-4.

10 Hanley JA, McNeil BJ. A method of comparing the areas under receiver operating characteristic curves derived from the same cases. Radiology $1983 ; 148: 839-43$

11 Bates D, Caronna JJ, Cartlidge N, Knill JR, Levy DE, Shaw DA, et al. A prospective study of nontraumatic coma: methods and results in 310 patients. Ann Neurol 1977;2:211-20.

12 Mills ML, Russo LS, Vines FS, Ross BA. High-yield criteria for urgent cranial computed tomography scans. Ann Emerg Med 1986;15:1167-72.

13 Swets JA. Measuring the accuracy of diagnostic systems. Science 1988;240:1285-93.

14 Levy LL, Wallace JD. Cerebral blood flow regulation. II. Vasodilator mechanisms. Stroke 1977;8:189-93.

15 Levy LL, Wallace JD, Stolwijk JA, Poindexter ER. Cerebral blood flow regulation: vascular resistance adjustments in the circle of Willis. Stroke $1976 ; 7: 147-50$.

(Accepted 26 April 2002) 